\title{
Working During Pandemic Disasters: Views and Predictors of EMS Providers
}

\author{
Mahmoud T. Alwidyan, PhD (10; Alaa O. Oteir, PhD; Joseph Trainor, PhD
}

\section{ABSTRACT}

Objectives: This study aims to assess the perception and attitude of emergency medical services (EMS) providers toward working during disease outbreaks, and the factors that may influence their decisions to ultimately work or not.

Methods: This is a cross-sectional study assessing the attitude of EMS providers to work during disease outbreaks. Descriptive statistics and regression analyses were performed to assess attitudes toward reporting for duty and factors that influence providers' decisions.

Results: Of the 500 surveys distributed, 466 (93.2\%) were complete and included for analysis. The majority of participants $(70.2 \%)$ are male with a mean age of 27 (SD 4.3 ) years. The study found that the majority $(71.1 \%)$ of participants are willing to come to work during disease outbreaks. The study found 7 predictors of reporting for duty. Confidence that employer will provide adequate protective gear was the most significant predictor (odds ratio [OR], 3.95; 95\% confidence interval $[\mathrm{Cl}]=2.31-5.42$ ). Concern for family safety was the most important barrier against coming to work $(\mathrm{OR}, 0.40 ; 95 \% \mathrm{Cl}=0.21-0.73$ ).

Conclusions: Providing adequate supplies of protective gear along with knowledge and training for disease outbreak are the main factors that enhance providers to fulfill their work expectations.

Key Words: civil defense, disaster medicine, disease outbreaks, emergency medical services, emergency nursing

E mergency medical services (EMS) providers give medical and trauma care to patients. They are the first line of care for those with urgent needs and often stabilize patients for transport to definitive care facilities. During disasters and public health emergencies, they also fill an integral role by supporting health care, public health, and public safety. ${ }^{1}$ In such situations, those first responders are at significant risk of injury and death. For instance, during the severe acute respiratory syndrome (SARS) outbreak in Toronto, Canada, in 2003, of the 850 paramedics who responded, more than half were exposed to SARS and placed in quarantine, some of them developed SARS-like symptoms, and 4 of them were hospitalized. ${ }^{1,2}$ This incident resulted in a dramatic decrease in their workforces, which negatively affected the health-care system in the area by reducing its surge capacity. Moreover, the recent Ebola outbreak in 2014 has shown high infection and death rates among health-care professionals. A report from the World Health Organization (WHO) shows that health-care workers are 21 to 32 times more likely to be infected with Ebola compared with the general population. ${ }^{3}$ These disease outbreaks resurfaced the concern of willingness of health-care professionals to work during such pandemics.
Generally, EMS providers, as a part of the health-care system, are willing to prioritize the needs of their patients over their personal needs and safety, especially during disasters. ${ }^{4}$ While EMS providers have ethical and professional duties to work, these duties have limits when doing so could put them or their family members in serious dangers. ${ }^{5}$ Research studies demonstrate different findings regarding the attitude of health-care workers to work during different types of disasters, including disease outbreaks. ${ }^{6-12}$ Studies found that man-made events and pandemics are typically the disasters to which first responders feel unfamiliar and fear, and in turn, are less willing to respond. ${ }^{13-15}$

During disasters, sufficient staffing is essential to keep the health-care system functional. EMS is an invaluable asset and considered the portal to the larger health-care system. ${ }^{1}$ In addition to their traditional work, during disease outbreaks, EMS providers can also help in other roles, such as distributing and administering vaccines and medications, as well as providing community education. ${ }^{1}$ While EMS personnel are among the frontline health-care providers during disasters, there is very little research on their attitude during disasters and public health emergencies. ${ }^{16}$ To the best of the researchers' knowledge, there is a lack of such 
research in Jordan and the Middle East. Therefore, the aim of this study was to assess the perception and attitude of EMS providers in Jordan to work during disease outbreaks, as well as the factors that may influence their decision.

\section{METHODS \\ Design/The Survey}

An expert panel developed a survey questionnaire specifically for EMS providers in Jordan. The paper-based survey was developed in English then translated into the Arabic language to make it more understandable by potential participants. The survey included 37 items addressing the following domains: (1) demographics (8 items); (2) attitude toward working during disease outbreaks (1 item); (3) concerns for working during disease outbreaks ( 7 items; Cronbach's alpha $=0.834$; low and high concern); (4) employer and the workplace (12 items; Cronbach's alpha $=0.826$; agree and disagree); (5) work obligation ( 8 items; Cronbach's alpha $=0.890$; obligated and not obligated); and (6) role of family ( 2 items). Factor analyses and internal reliability analyses were performed whenever appropriate to reduce variables and validate categories. ${ }^{17}$

To examine the attitude, a disease outbreak scenario was developed. The scenario is about a disease outbreak unfolding in a country outside Jordan with very limited information about its characteristics. Early reports show that it is an airborne disease with flu-like symptoms and high mortality rates. Some cases of the disease were reported in Jordan, and eventually in the workplace. Participants were then asked about their response to such a situation. They were asked to choose the most appropriate choice from the given five alternatives. These alternatives were: (1) turn off my cell phone so I will not be asked to come to work; (2) I'm not coming to work until I'm clear about the exposure risk; (3) only work my scheduled shifts; (4) I will work additional shifts if asked; (5) I will call my supervisor to ask if they need a shift covered.

The third to sixth domains are 6-point Likert-type questions. The questions outlined a series of statements related to work during disease outbreaks. Participants were asked to choose from 1 to 6 with 1 represents "strongly disagree" and 6 represents "strongly agree." Other choices for the domains include "not at all concerned" and "extremely concerned"; "not at all confident" and "extremely confident", and "not at all obligated" and "extremely obligated".

\section{Setting}

EMS system in Jordan is exclusively provided by the Jordanian Civil Defense (JCD), a quasi-military system. EMS in Jordan comprises of approximately 2000 providers that encompass emergency medical technicians (EMTs), intermediate, and paramedics who provide all types of prehospital care.

\section{Participants}

The questionnaire was pilot tested on 10 participants, and was then modified according to the feedback provided. The expert panel approved the final form of the survey. Over the months of October and November 2018, a total of 500 surveys were distributed randomly to frontline EMS providers in the JCD. The surveys were handed to the EMS director who distributed them evenly to the EMS departments and stations across the country. Each department was informed by the EMS director to give the chance to all ambulance workers to voluntarily participate in the study. The number of questionnaires was stratified according to the number of EMS workers in the regions (north, middle, and south) to give equal chance for all potential participants. Completed surveys were returned in sealed envelopes to Jordan University of Science and Technology (JUST) for further data entry and analyses.

\section{Data Analysis}

To begin analysis, all continuous variables were summarized as means and standard deviation (SD) and categorical variables were reported as frequencies and percentages. Participant's responses were dichotomized where possible to indicate positive or negative attitude. For instance, in the 6-point Likerttype questions, the first 3 choices were merged and considered "disagree," a negative attitude; and the last 3 choices were merged and considered "agree," a positive attitude. In addition, for the scenario question, choices were also dichotomized into "willing" and "unwilling" to allow for binary logistic regression.

Bivariate regression analyses were individually conducted to all independent variables to assess the impact of each variable on the likelihood to report for duty during a disease outbreak. Variables with a $P$ value less than 0.1 were entered into the full model. Binary logistic regression was then conducted using a backward stepwise method to identify the predictors of willingness to report for duty, with a $\mathrm{P}$-value of 0.05 to determine the statistical significance. All analyses were performed using SPSS version 22 (Chicago, IL).

\section{Ethical Approval}

JUST Institutional Review Board (IRB) reviewed and approved the study procedure before starting data collection (IRB NO: 24/113/2018). The research team also contacted the JCD regarding the survey and obtained the approval.

\section{RESULTS}

Of 500 surveys distributed, 34 surveys had missing information and were excluded from the study, accounting for these exclusions the dataset included 466 (93.2\%) completed surveys. All participants currently working in the JCD and completed the survey were included in the study. Table 1 describes the demographics of the study participants. The participants had a mean age of 27 (SD 4.3) years; the majority were males 


\begin{tabular}{|llc|}
\hline \multicolumn{1}{|c|}{ Demographics of the Study Participants } \\
Variable & \multicolumn{1}{c|}{$\mathbf{n}(\%)$} \\
Gender & Male & $325(70.2)$ \\
& Female & $138(29.8)$ \\
Age (years) & Mean (SD) & $27.85(4.382)$ \\
Marital status & Single & $182(39.8)$ \\
& Married & $275(60.2)$ \\
Have children & Yes & $234(51.5)$ \\
& No & $220(48.5)$ \\
Education & High school & $142(30.7)$ \\
& Diploma & $275(59.5)$ \\
Job title (or & Bachelors or above & $45(9.7)$ \\
certification) & EMT & $43(9.4)$ \\
& EMT-intermediate & $86(18.7)$ \\
Experience (years) & Paramedic & $330(71.9)$ \\
& Mean (SD) & $8.45(4.170)$ \\
\hline
\end{tabular}

Abbreviations: EMT, emergency medical technician; SD, standard deviation $\mathrm{N}=466$.

(70.2\%). The majority were certified as paramedics (71.9\%), with a mean work experience of 8 years (SD 4.1).

\section{Participants' Perception Toward Working in a Hypothetical Pandemic Scenario}

Table 2 summarizes the participants' responses to the hypothetical outbreak scenario. Responses demonstrated that "sticking with the scheduled shifts" is the option that participants chose the most (24.1\%), and the option chosen the least was "turning off cell phone" (8.7\%). Responses were dichotomized into "not willing" and "willing" with the majority of participants $(71.1 \%)$ are willing to report for duty in the case of a disease outbreak (Table 2).

\section{Factors Influencing Reporting to Work During Disease Outbreaks}

Survey participants were asked about their concerns toward work during disease outbreaks such as the given scenario. More than two-thirds of participants (68.5\%) expressed concern of "becoming infected and getting ill," $67.3 \%$ were concerned about "dying from infection," and, of interest, 79.0\% were concerned about "infecting family members." Threequarters $(75.2 \%)$ of participants were concerned from the "lack of appropriate information" about the outbreak, 70.4\% concerned about the "shortage in personal protective equipment" (PPE), and 79.8\% concerned from "lack of availability of vaccines or effective treatment." Overall, $72.7 \%$ of participants expressed their concern about working during disease outbreaks.

Three-quarters $(75.1 \%)$ of participants agree that "disease outbreaks put their families at risk of infection higher than the general population," and $60.3 \%$ agree that their "concern for family has a major effect on their decision to come to work."
When it comes to "who comes first during disease outbreaks," work obligation was indicated the highest $(45.3 \%)$, followed by family safety (29.2\%), and self-safety (25.5\%).

Participants were also asked about their employer and the workplace. More than half of the study participants (56.3\%) agree that their employer has "efficient systems in place to manage disease outbreaks," $61.1 \%$ agree that their employer will provide "updated information" about the progress of the outbreak and "adequate" PPE, 63.4\% agree that their employer will provide "treatment and vaccines" once available. Overall, more than two-thirds $(68.5 \%)$ of participants were confident that their employer would perform their responsibilities to keep workers safe. With regard to disciplinary actions, $61.3 \%$ of participants agree that their employer will implement strict disciplinary actions against workers who did not come to work during disease outbreaks, and less than half (40.9\%) agree that they are coming to work because of these disciplinary actions. Yet, less than half $(42.6 \%)$ believe that those who did not show up to work should be punished.

When it comes to knowledge and training, more than twothirds $(68.1 \%)$ of participants agree that they have "adequate knowledge and training" for disease outbreaks, and 59\% believe that "lack of knowledge and training could influence their decision to come to work."

\section{Predictors of Reporting for Duty During Disease Outbreaks}

As shown in Table 3, demographic characteristics were examined for their influence on reporting for duty using bivariate regression analyses. Age (bivariate odds ratio [OR], 0.98; $95 \%$ confidence interval $[\mathrm{CI}]=0.94-1.03$ ), gender (bivariate $\mathrm{OR}, 0.81 ; 95 \% \mathrm{CI}=0.52$ to 1.25 ), marital status (bivariate $\mathrm{OR}, 0.96 ; 95 \% \mathrm{CI}=0.63$ to 1.46 ), presence of children (bivariate $\mathrm{OR}, 1.26 ; 95 \% \mathrm{CI}=0.83$ to 1.91 ), and years of experience (bivariate $\mathrm{OR} 0.98 ; 95 \% \mathrm{CI}=0.93$ to 1.04 ) were not significant predictors of reporting for duty. While education (diploma) and job title (EMT-intermediate) show significance on the bivariate regression analyses (bivariate $\mathrm{OR}, 2.68$; $95 \% \mathrm{CI}=1.15$ to 6.19 ; bivariate $\mathrm{OR}, 2.35 ; 95 \% \mathrm{CI}=1.21$ to 4.56 , respectively), they did not show significance in the multivariate model.

Yet, seven independent variables remained statistically significant in the multivariate logistic regression model. Respondents who are confident that employer will provide adequate PPE (OR, 3.95; 95\% CI = 2.31-5.42), have adequate knowledge and training for disease outbreaks (OR, 3.04; 95\% $\mathrm{CI}=1.71-5.39$ ), agree with the strict disciplinary actions to enforce reporting for duty (OR, 2.52; 95\% $\mathrm{CI}=1.33-4.78)$, or feel obligated to work even if some co-workers become infected with the disease $(\mathrm{OR}, 2.19 ; 95 \% \mathrm{CI}=1.15-4.18)$ were significantly more likely to report for duty during disease outbreaks. Conversely, those who feel obligated to work if they did 


\begin{tabular}{|c|c|c|c|c|}
\hline \multicolumn{5}{|c|}{ Frequencies of Participants for Scenario Options } \\
\hline 1 & $\begin{array}{l}\text { Turn off my cell phone so I will } \\
\text { not be asked to come to work. }\end{array}$ & $40(8.7)$ & Not willing & $133(28.9)$ \\
\hline 2 & $\begin{array}{l}\text { I'm not coming to work until I'm } \\
\text { clear about the exposure risk. }\end{array}$ & $93(20.2)$ & & \\
\hline 3 & Only work my scheduled shifts. & $111(24.1)$ & Willing & $327(71.1)$ \\
\hline 5 & $\begin{array}{l}\text { I will call my supervisor to ask if } \\
\text { they need a shift covered. }\end{array}$ & 107 (23.3) & & \\
\hline
\end{tabular}

$\mathrm{N}=460$.

\section{TABLE 3}

\section{Predictors of Reporting for Duty}

\section{Predictor}

Age

Gender

Marital status

Presence of children

Years of experience

Education: High school

Diploma

Bachelor's or above

Job title: EMT

EMT intermediate

Paramedic

Confidence that employer will provide adequate PPE (Yes)

I have adequate knowledge

and training for disease

outbreaks (Yes)

EMS providers not showing

up during disease

outbreaks should receive

strict disciplinary actions

(Yes)

Work obligation if some

coworkers got infected with

the disease (yes)

Work obligation if you did not receive appropriate training (yes)

Family concern has a major effect on my decision to come to work (yes)

Concerned about shortage in PPE (Yes)

\section{Bivariate OR \\ (95\% Cl)}

0.98 (0.94 to 1.03$)$

$0.81(0.52$ to 1.25$)$

0.96 (0.63 to 1.46$)$

1.26 (0.83 to 1.91$)$

0.98 (0.93 to 1.04$)$

2.68 (1.15 to 6.19$)$

1.72 (0.76 to 3.88 )

2.35 (1.21 to 4.56$)$

1.02 (0.60 to 1.76 )

3.54 (2.31 to 5.42 )

4.57 (2.95 to 7.07 )

4.13 (2.57 to 6.65 )

$<0.001^{*}$

$<0.001^{*}$

2.19 (1.15 to 4.18$)$

$0.017^{*}$

1.55 (1.01 to 2.40 )

$0.044^{*}$

$0.52(0.27$ to 0.99$)$

0.049 *

0.45 (0.28 to 0.70$)$

$0.001^{*}$

$0.40(0.21$ to 0.73$)$

$0.003^{*}$

0.56 (0.34 to 0.90$)$

$0.019 *$

$0.40(0.20$ to 0.76$)$

$0.006^{*}$

Abbreviations: CI, confidence interval; EMS, emergency medical services; EMT, emergency medical technician; OR, odds ratio; PPE, personal protective equipment. *Significant $P$ value.

not receive appropriate training $(\mathrm{OR}, 0.52 ; 95 \% \mathrm{CI}=0.27$ 0.99), agree with the family effect on their decision to come to work (OR, 0.40; 95\% $\mathrm{CI}=0.21-0.73$ ), or concerned about shortage in PPE (OR, 0.40; 95\% CI =0.20-0.76) were significantly less likely to report for duty during disease outbreaks.

\section{DISCUSSION}

Our findings indicate that in a scenario of a disease outbreak, more than two-thirds of the study participants would be willing to come to work. Previous research studies are diverse in their results. That is, in a systematic review, Connor (2014) found 
that health-care professionals are $25 \%$ to $82 \%$ willing to work during pandemics compared with $83 \%$ to $90 \%$ for natural disasters. ${ }^{13}$ In the EMS field, Tippett et al. (2010) found that $56.3 \%$ of the study sample are willing to work during outbreak conditions. ${ }^{18}$ In another study, Barnett et al. (2010) showed more optimistic results as they found that $93 \%$ of EMS professionals would be willing to report to work if required, and $88 \%$ if asked, but not required. ${ }^{19}$ Of interest, Alwidyan (2016) found that, while the interviewed EMS participants were "thrilled" and "excited" to work during natural disasters, they expressed varying concerns about working in pandemic conditions. ${ }^{20}$

\section{Predictors of Reporting for Duty}

The study participants were diverse in their age, gender, marital status, presence of children, education, job title, and experience. These differences were examined for their potential effect on the decision to reporting for duty. The study found that, except for education and job title, demographics were not predictors of reporting for duty during disease outbreaks. This contradicts with previous research studies indicating that male gender and prior experience are predictors of willingness to come to work, while age reported having contradictory findings. ${ }^{7}$ Our study found, however, that those who have diploma degree or being EMT-intermediate were predictors on the bivariate analyses, but were not remained on the multivariate logistic model (Table 3).

Among the 23 independent variables included in the final model, 7 variables remained significant predictors on the multivariate logistic regression. Confidence that employer will provide adequate $\mathrm{PPE}$, having adequate knowledge and training for disease outbreaks, agreement with strict disciplinary actions against providers who did not show up during disease outbreaks, and feeling obligated to work even if some coworkers become infected were predictors of reporting for work. On the other hand, not receiving appropriate training, concern for family, and concern about shortage in PPE were predicted barriers of reporting for work.

It is reported that confidence in the employer was a predictor of willingness to work during disasters. ${ }^{12}$ Given that disease outbreaks are associated with a high level of uncertainty in the early stages, employers need to communicate with front-line workers and keep them abreast of the evolving outbreak. ${ }^{21}$ Our study found that more than two-thirds of participants were confident that their employer would perform their responsibilities to keep workers safe. Additionally, the study found that the majority $(61.1 \%)$ of participants were confident that their employer will provide adequate supplies of PPE. Those who were confident with their employer to provide PPE were 4 times more likely to report to work than those who lack confidence (Table 3). Conversely, the present study found that concerns for shortage in PPE found to be a major predicted barrier for reporting to work, which is congruent with the findings of previous research studies. ${ }^{19,22}$

More than two-thirds of the study participants indicated that they have appropriate knowledge and training for disease outbreaks. Those who indicated that they have adequate knowledge and training are 3 times more likely to come to work during disease outbreaks as indicated by the logistic regression model (Table 3). Not receiving appropriate training, however, was found to be a predicted barrier for coming to work. These results are in congruence with a previous study on paramedics indicating that willingness to work increase significantly with adequate knowledge about the disease. ${ }^{18}$ It is worth mentioning here that EMS providers are not trained to diagnose infections in the prehospital settings, rather they are trained to appropriately handle patients with potential infections. ${ }^{23}$ Nevertheless, a study by Shaban (2006) found that EMS personnel have poor knowledge of infectious diseases and principles of infection control. ${ }^{24}$

Concerns for family have a major effect on reporting to work as indicated by the study findings. Participants indicated that they are concerned about infecting their family members (79.0\%) more than their concern of becoming infected themselves $(68.5 \%)$, which was also reported by previous research. $8,13,20,21$ Our study also found that the majority of participants $(60.3 \%)$ indicated that their family has a major effect on their decision to come to work, and this was the strongest predicted barrier on reporting to work as indicated by the logistic regression model (Table 3 ). These findings are in congruence with the previous studies. According to Trainor and Barsky (2011), the main source of role conflict during disasters is the uncertainty regarding the safety of family members and the feeling that first responders should protect their families. ${ }^{12}$ Other research studies show that family safety was number one concern for all health-care professionals during disease outbreaks. ${ }^{19,21,22}$ For instance, although Mackler et al. (2007) found that $91 \%$ of the participants would remain on duty if they have been vaccinated, this percentage falls to $38 \%$ if their families have not received the vaccine. ${ }^{22}$

This study provides one of the first empirical examinations of the potential behavior of EMS providers toward working during disease outbreaks in Jordan, and probably the Middle East. EMS employers need to know their potential workforce, the expected number of personnel who may, or may not, show up, and the factors that led to their absenteeism. Knowing the intended behavior of EMS providers enables decision-makers to plan for them and to implement measures that enhance willingness to report for duty. EMS needs to be efficient when it comes to plan for, respond to, and recover from health disasters.

\section{Limitations}

There are several limitations need to be discussed here. The study conducted on EMS providers of the Jordanian Civil 
Defense. While the sample size was more than $20 \%$ of the total EMS population and fairly representative in terms of demographics, we cannot exclude selection bias. That is, while the survey distribution was clustered according to Civil Defense departments and stations, there was no control over giving equal chance for all potential participants due to the quasi-military style of the Civil Defense in Jordan. Therefore, the findings of this study should be considered with caution. It is worth mentioning here that EMS in Jordan is much like the fire-based EMS, which is the most common in the United States and represents approximately $40 \%$ of all EMS agencies. ${ }^{25}$

The study looks for the potential attitude of people after receiving a hypothetical situation, an approach called perception studies. ${ }^{12}$ In real disease outbreaks; however, there will be many coincidental situations for each individual in the EMS system that the researcher cannot bring about in a simple case scenario. Therefore, this approach has difficulty in simulating the real-life situations of participants, which ultimately could affect the real beliefs and views of them. However, with little, if any, previous disease outbreak experience, it is challenging to anticipate with confidence the expected behavior of EMS participants, making perception studies the appropriate methodology to explore pandemic disasters.

\section{CONCLUSIONS}

This study found that even with the quasi-military style of EMS, approximately one-third of EMS providers may not show up if they believe that they or their family members become at risk of infection. The strict disciplinary actions by the authoritative EMS system was not a factor to enhance reporting for duty. Rather, confidence in employer of providing adequate supplies of PPE and vaccines as well as adequate knowledge and training for disease outbreak are the main predictors of willingness to come and work during pandemics. On the other hand, concerns for family safety and shortage in PPE found to be the major barriers for fulfilling their duties.

Continuing education courses about infectious diseases and infection control could be the starting point for improving the EMS workforces during pandemics. Equally important, the EMS employers need to provide appropriate supplies of PPE and the training on their use such as donning and doffing procedures. This would protect providers from the risk of infection, ameliorate their concerns of infection, and enhance their trust relationship with their employers.

\section{About the Authors}

Paramedics Program, Department of Allied Medical Sciences, Faculty of Applied Medical Sciences, Jordan University of Science and Technology, Irbid, Jordan (Drs Alwidyan, Oteir); Department of Paramedicine, Monash University,
Melbourne, Victoria, Australia (Dr Oteir) and Disaster Research Center, Biden School of Public Policy and Administration, University of Delaware, Newark, Delaware (Dr Trainor).

Correspondence and reprint requests to Mahmoud T. Alwidyan, Paramedics Program, Department of Allied Medical Sciences, Faculty of Applied Medical Sciences, Jordan University of Science and Technology, Irbid, Jordan (e-mail: mtalwidyan@just.edu.jo).

\section{Funding}

This project was funded by Jordan University of Science and Technology (Research grant number: 20180092).

\section{REFERENCES}

1. Maguire BJ, Dean S, Bissell RA, et al. Epidemic and bioterrorism preparation among emergency medical services systems. Prehosp Disaster Med. 2007;22(3):237-242. doi: 10.1017/S1049023X0000474X

2. Silverman A, Simor A, Loutfy MR. Toronto Emergency Medical Services and SARS. Emerg Infect Dis. 2004;10(9):1688-1689.

3. WHO. Health Worker Ebola Infections in Guinea, Liberia and Sierra Leone. 2015;(May):1-16. http://www.who.int/hrh/documents/21may2015_ web_final.pdf. Accessed June 17, 2020.

4. Chaffee M. Willingness of health care personnel to work in a disaster: an integrative review of the literature. Disaster Med Public Health Prep. 2009;3:42-65. doi: 10.1007/s13398-014-0173-7.2

5. Singer PA, Benatar SR, Bernstein M, et al. Ethics and SARS: lessons from Toronto. BMJ. 2003;327(7427):1342-1344. doi: 10.1136/bmj.327.7427. 1342

6. Damery S, Draper H, Wilson S, et al. Healthcare workers' perceptions of the duty to work during an influenza pandemic. J Med Ethics. 2010;36(1):12-18. doi: 10.1136/jme.2009.032821

7. Devnani M. Factors associated with the willingness of health care personnel to work during an influenza public health emergency: an integrative review. Prehosp Disaster Med. 2012;27(6):551-566. doi: 10.1017/ S1049023X12001331

8. Dimaggio C, Markenson D, Loo GT, et al. The willingness of U.S. Emergency Medical Technicians to respond to terrorist incidents. Biosecur Bioterror. 2005;3(4):331-337. doi: 10.1089/bsp.2005.3.331

9. Draper H, Wilson S, Ives J, et al. Healthcare workers' attitudes towards working during pandemic influenza: a multi method study. BMC Public Health. 2008;8:192. doi: 10.1186/1471-2458-8-192

10. Kohloff SA, Crouch B, Robin PM, et al. Evaluation of hospital mass screening and infection control practices in a pandemic influenza full-scale exercise. Disaster Med Public Health Prep. 2012;6:378-384.

11. Qureshi K, Gershon RRM, Sherman MF, et al. Health care workers' ability and willingness to report to duty during catastrophic disasters. J Urban Health. 2005;82(3):378-388. doi: 10.1093/jurban/jti086

12. Trainor J, Barky L. Reporting For Duty? A Synthesis of Research on Role Conflict, Strain, and Abandonment Among Emergency Responders During Disasters and Catastrophes. Newark, DE: University of Deleware; 2011.

13. Connor SB. When and why health care personnel respond to a disaster: the state of the science. Prehosp Disaster Med. 2014;29(3):270-274. doi: $10.1017 /$ S1049023X14000387

14. Gershon RRM, Vandelinde N, Magda LA, et al. Evaluation of a pandemic preparedness training intervention of emergency medical services personnel. Prehosp Disaster Med. 2009;24(6):508-511. doi: 10.1017/ S1049023X00007421

15. Smith E, Morgans A, Qureshi K, et al. Paramedics' perceptions of risk and willingness to work during disasters. Aust J Emerg Manag. 2009;24(3):21-27.

16. Watt K, Tippett VC, Raven SG, et al. Attitudes to living and working in pandemic conditions among emergency prehospital medical care 
personnel. Prehosp Disaster Med. 2010;25(1):13-19. doi: 10.1017/ S1049023X00007597

17. Pallant J. SPSS Survival Manual. A Step by Step Guide to Data Analysis Using SPSS. 4th ed. Crows Nest, Australia: Allen \& Unwin; 2011.

18. Tippett VC, Watt K, Raven SG, et al. Anticipated behaviors of emergency prehospital medical care providers during an influenza pandemic. Prehosp Disaster Med. 2010;25(1):20-25. doi: 10.1017/S10490 23X00007603

19. Barnett DJ, Levine R, Thompson CB, et al. Gauging U.S. Emergency Medical Services workers' willingness to respond to pandemic influenza using a threat-and efficacy-based assessment framework. PLoS One. 2010;5(3):e9856. doi: 10.1371/journal.pone.0009856

20. Alwidyan MT. Reporting for duty during disease outbreaks. Nat Hazards Cent. 2016;XL(8). https://hazards.colorado.edu/article/reporting-forduty-during-disease-outbreaks-the-views-of-emergency-medical-serviceproviders. Accessed June 17, 2020.
21. Ives J, Greenfield S, Parry JM, et al. Healthcare worker's attitudes to working during pandemic influenza: a qualitative study. BMC Public Health. 2009;9(1):56. doi: 10.1186/1471-2458-9-56

22. Mackler N, Wilkerson W, Cinti S. Will first-responders show up for work during a pandemic? Lessons from a smallpox vaccination survey of paramedics. Disaster Manag Response. 2007;5:45-48. doi: 10.1016/j.dmr. 2007.02.002

23. Bissell RA, Seaman KG, Bass RR, et al. Change the scope of practice of paramedics? An EMS/public health policy perspective. Prehosp Emerg Care. 1999;3(2):140-149. doi: 10.1080/10903129908958923

24. Shaban RZ. Paramedic knowledge of infection control principles and standards in an Australian emergency medical system. Healthc Infect. 2006;11(1):13. doi: 10.1071/HI06013

25. NHTSA. Traffic safety facts: EMS system demographics. 2014. http:// www.ems.gov/pdf/812041-Natl_EMS_Assessment_2011.pdf. Accessed June 17, 2020. 